\title{
Multiple hepatocyte nuclear factor 1A (HNF1A)-inactivated hepatocellular adenomas arising in a background of congenital hepatic fibrosis
}

\author{
Yangkyu Lee ${ }^{1}$, Hyunjin Park ${ }^{2}$, Kyoungbun Lee ${ }^{3}$, Youngeun Lee ${ }^{3}$ Kiryang Lee ${ }^{3}$, Haeryoung Kim ${ }^{3}$ \\ 'Department of Pathology, Seoul National University Bundang Hospital, Seoul National University College of Medicine, Seongnam; \\ 2Department of Pathology, Gangnam Severance Hospital, Yonsei University College of Medicine, Seoul; \\ ${ }^{3}$ Department of Pathology, Seoul National University Hospital, Seoul National University College of Medicine, Seoul, Korea
}

Hepatocellular adenomas (HCAs) are benign hepatocellular neoplasms with distinct molecular subtypes [1,2]. Hepatocyte nuclear factor 1A (HNF1A)-inactivated hepatocellular adenoma (H-HCA) occupies about $35 \%$ of all HCAs [1,2]. H-HCA demonstrates somatic $(90 \%)$ or germline $(10 \%)$ inactivating mutations of HNF1A gene, which encodes a transcription factor involved in several metabolic pathways of hepatocytes, and inactivation of HNF1A promotes lipogenesis [3]. Clinically, HHCAs occur predominantly in females, are frequently associated with oral contraceptives, and a subset of H-HCA with germline HNF1A mutations is associated with maturity-onset diabetes type 3 (MODY3). Morphologically, H-HCAs frequently show steatosis and loss of liver fatty acid-binding protein (LFABP) expression in the tumor cells $[1,2]$.

Here, we report a rare case of a young male patient with multiple H-HCAs in a background of congenital hepatic fibrosis (CHF).

\section{CASE REPORT}

A 25-year-old man was admitted to the hospital for liver transplantation for multiple hepatic masses that were incidentally discovered by imaging. He had previously been diagnosed with CHF on liver biopsy 14 years ago, and had received transjugular intrahepatic portosystemic shunt for portal hypertension at that time. He had no history of metabolic syndrome, al-

Received: September 15, 2020 Revised: November 9, 2020

Accepted: November 12, 2020

Corresponding Author: Haeryoung Kim, MD, PhD

Department of Pathology, Seoul National University Hospital, Seoul National University

College of Medicine, 103 Daehak-ro, Jongno-gu, Seoul 03080, Korea

Tel: +82-2-740-8322, Fax: +82-2-765-5600, E-mail: haeryoung.kim@snu.ac.kr cohol intake or prior intake of androgens and his body mass index was $25.2 \mathrm{~kg} / \mathrm{m}^{2}$. There was no evidence of other abnormalities associated with CHF, and there was no remarkable family history. Preoperative liver function tests were as follows: aspartate aminotransferase $35 \mathrm{U} / \mathrm{L}$, alanine aminotransferase 29 U/L, gamma-glutamyl transferase $19 \mathrm{U} / \mathrm{L}$, alkaline phosphatase $79 \mathrm{U} / \mathrm{L}$, total bilirubin $4.2 \mathrm{mg} / \mathrm{dL}$, and direct bilirubin $1.3 \mathrm{mg} / \mathrm{dL}$. Serological tests for hepatitis B and C virus were negative. On computed tomography, there were multiple fat-containing nodules in both hepatic lobes.

The liver weighed $1,242 \mathrm{~g}$, and sections revealed nine discrete tan-to-yellow nodules in both lobes, measuring $9.5 \times 6.5 \times 7.5 \mathrm{~cm}$ in the largest one. The background liver was firm with a reticular appearance (Fig. 1). On microscopic examination, the background liver showed the typical histological features of CHF: broad fibrous bands rimmed by small bile ductular structures with ductal plate malformation patterns (Fig. 1). The pathological findings of the nine nodules are summarized in Table 1 and Figs. 2 and 3. Six nodules were diagnosed as H-HCAs and the three smaller nodules as large regenerative nodule (LRN) or focal nodular hyperplasia (FNH)-like nodules, based on the histopathological and immunohistochemical characteristics. Notably, although LFABP loss was seen in all six H-HCAs, nodules \#1, \#2, and \#9 showed only patchy mild steatosis, and nodules \#1 and \#2 also demonstrated focal peliosis and mild ductular reaction. The remaining $4 \mathrm{H}-\mathrm{HCAs}$ demonstrated diffuse steatosis. On follow-up, the patient is currently well without evidence of recurrence.

\section{DISCUSSION}

To our knowledge, this is the second reported case of $\mathrm{H}-$ 
HCA arising in a background of CHF. Hepatocellular nodules, including FNH and FNH-like nodules, HCAs and even hepatocellular carcinomas (HCCs), have been demonstrated to occur in the setting of hepatic vascular abnormalities, such as BuddChiari syndrome and congenital extrahepatic portosystemic shunts [4-8]. While CHF belongs to a group of fibropolycystic diseases of the liver that affects the biliary system, portal hypertension dominates the clinical presentation of this disease, as the number of portal vein branches is often reduced [9]. Treatment of CHF with portosystemic shunt surgery may also add to the
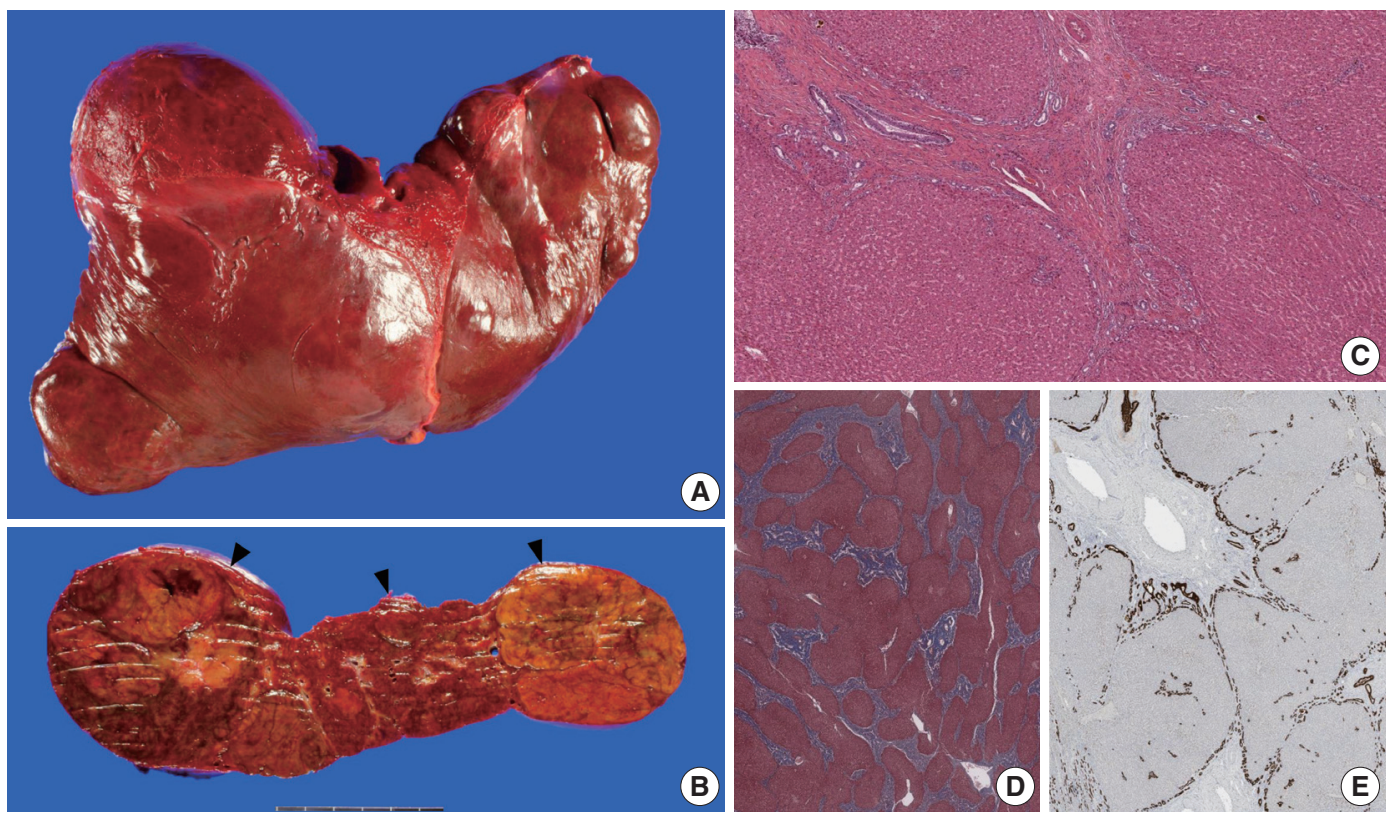

Fig. 1. (A-C) Gross findings. (A) The explanted liver is distorted in shape due to the multiple bulging masses. (B) A representative section reveals three lobulated nodules (arrowheads). The nodules are lobulated, well-demarcated but non-encapsulated, with focal hemorrhage and peliosis. (C) On microscopy, the background liver shows broad fibrous bands lined by ductular structures with ductal plate malformation pattern and inspissated bile. (D) Masson's trichrome stain reveals the jigsaw-puzzle pattern biliary fibrosis. (E) Cytokeratin 7 immunostain highlights the ductular structures at the interface between the fibrous bands and the hepatic lobules.

Table 1. Summary of the pathological features of the hepatic nodules in this case

\begin{tabular}{|c|c|c|c|c|c|}
\hline No. & $\begin{array}{l}\text { Location } \\
\text { (segment) }\end{array}$ & Size (cm) & Diagnosis & Histopathological feature & Immunohistochemical stain result \\
\hline 1 & S7 & 9.5 & $\mathrm{H}-\mathrm{HCA}$ & $\begin{array}{l}\text { Patchy steatosis, focal peliosis, } \\
\text { bile ductules }\end{array}$ & $\begin{array}{l}\text { LFABP loss, SAA/CRP/GS negative, membranous } \beta \text {-catenin } \\
\text { expression }\end{array}$ \\
\hline 2 & S6 & 6.6 & $\mathrm{H}-\mathrm{HCA}$ & $\begin{array}{l}\text { Patchy steatosis, focal peliosis, } \\
\text { bile ductules }\end{array}$ & $\begin{array}{l}\text { LFABP loss, SAA/CRP/GS negative, membranous } \beta \text {-catenin } \\
\text { expression }\end{array}$ \\
\hline 3 & S1 & 3.5 & $\mathrm{H}-\mathrm{HCA}$ & Diffuse steatosis & $\begin{array}{l}\text { LFABP loss, SAA/CRP/GS negative, membranous } \beta \text {-catenin } \\
\text { expression }\end{array}$ \\
\hline 4 & S4 & 4.5 & $\mathrm{H}-\mathrm{HCA}$ & Diffuse steatosis & $\begin{array}{l}\text { LFABP loss, SAA/CRP/GS negative, membranous } \beta \text {-catenin } \\
\text { expression }\end{array}$ \\
\hline 5 & S4 & 1.0 & LRN & Fibrous septa with bile ductules & $\begin{array}{l}\text { No LFABP loss, focal SAA/CRP expression, GS negative, } \\
\text { membranous } \beta \text {-catenin }\end{array}$ \\
\hline 6 & S4 & 1.0 & FNH-like nodule & Fibrous septa with bile ductules & $\begin{array}{l}\text { No LFABP loss, focal SAA/CRP expression, patchy GS } \\
\text { expression, membranous } \beta \text {-catenin }\end{array}$ \\
\hline 7 & S2 & 9.5 & $\mathrm{H}-\mathrm{HCA}$ & Diffuse steatosis & $\begin{array}{l}\text { LFABP loss, SAACRP/GS negative, membranous } \beta \text {-catenin } \\
\text { expression }\end{array}$ \\
\hline 8 & S2 & 0.9 & FNH-like nodule & Fibrous septa with bile ductules & $\begin{array}{l}\text { No LFABP loss, focal SAA/CRP expression, patchy GS } \\
\text { expression, membranous } \beta \text {-catenin }\end{array}$ \\
\hline 9 & S6 & 1.6 & $\mathrm{H}-\mathrm{HCA}$ & Focal mild steatosis & $\begin{array}{l}\text { LFABP loss, SAA/CRP/GS negative, membranous } \beta \text {-catenin } \\
\text { expression }\end{array}$ \\
\hline
\end{tabular}

H-HCA, Hepatocyte nuclear factor 1A-inactivated hepatocellular adenoma; LFABP, liver fatty acid-binding protein; SAA, serum amyloid A; CRP, C-reactive peptide; GS, glutamine synthetase; LRN, large regenerative nodule; FNH-like nodule, focal nodular hyperplasia-like nodule. 

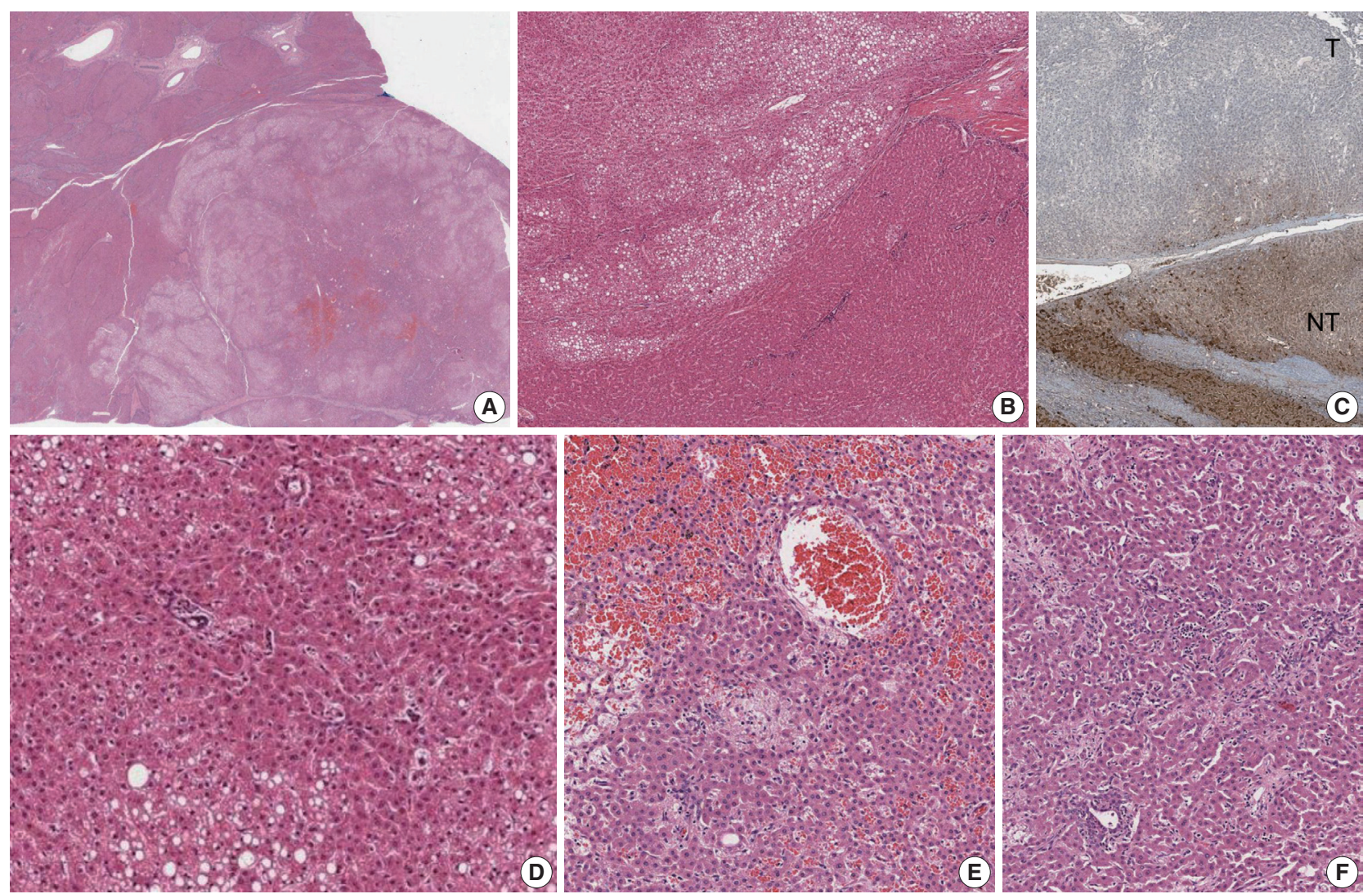

Fig. 2. The histopathological and immunohistochemical features of this case. (A) Scanning power view demonstrates a well-demarcated and non-encapsulated solid mass (lower right). (B) The peripheral portion of the tumor shows patchy macrovesicular steatosis. (C) Loss of liver fatty acid-binding protein expression is seen in the tumor (T, tumor; NT, non-tumor). (D) Higher power magnification of the hepatocellular adenoma, demonstrating the patchy macrovesicular steatosis and unpaired arteries. (E) No significant cytoarchitectural atypia is present. The central portion of nodule \#1 demonstrates focal sinusoidal dilatation with congestion and peliosis. (F) A few pseudoportal tracts are seen in nodule \#1, containing clusters of bile ductules.

abnormal heterogeneous vascularity in the parenchyme [10]. As a result, the uneven hepatic perfusion may give rise to the formation of hepatocellular nodules. However, although this vascular concept explains the pathogenesis of reactive hyperplastic hepatocellular nodules such as FNH and LRN/FNH-like nodules, the relationship between this vascular abnormality and the occurrence of neoplastic hepatocellular nodules is still not well understood. Indeed, the neoplastic nature of smaller hepatocellular nodules resembling HCAs (previously referred to as "adenoma-like" nodules or adenomatous hyperplasia) arising in vascular disorders have been questioned, due to the morphological overlap with LRNs and FNH-like nodules and their frequent co-existence within the same liver [4]. However, as the molecular classification of HCAs has been better characterized over the past decade, immunohistochemical stains have facilitated the diagnosis of HCAs [7]. Interestingly, Sempoux et al. [7] described three cases of H-HCAs arising in backgrounds of hepatic vascular disorders which were either associated with HCCs or contained atypical foci. This is a notable finding as it implies that H-HCAs associated with vascular disorders may be at increased risk of malignant transformation, in contrast to those arising in "conventional" settings (e.g., females, oral contraceptives, MODY3, etc) [2]. Another interesting finding is that H-HCAs in the context of hepatic vascular disorders often demonstrated a lack of intratumoral steatosis, which differs from the characteristic histology (diffuse steatosis) of typical H-HCAs [7]. Indeed, in our case, three HHCAs demonstrated only focal mild steatosis, and the diagnosis was rendered based on the loss of LFABP expression.

Although further validation would be required, the identification of nodular lesions on imaging in the liver of patients with CHF or hepatic vascular disorders may warrant histological examination to rule out the possibility of HCCs or HCAs. In addition, H-HCAs arising in the context of hepatic vascular disorders may not be associated with the typical clinicopathological fea- 

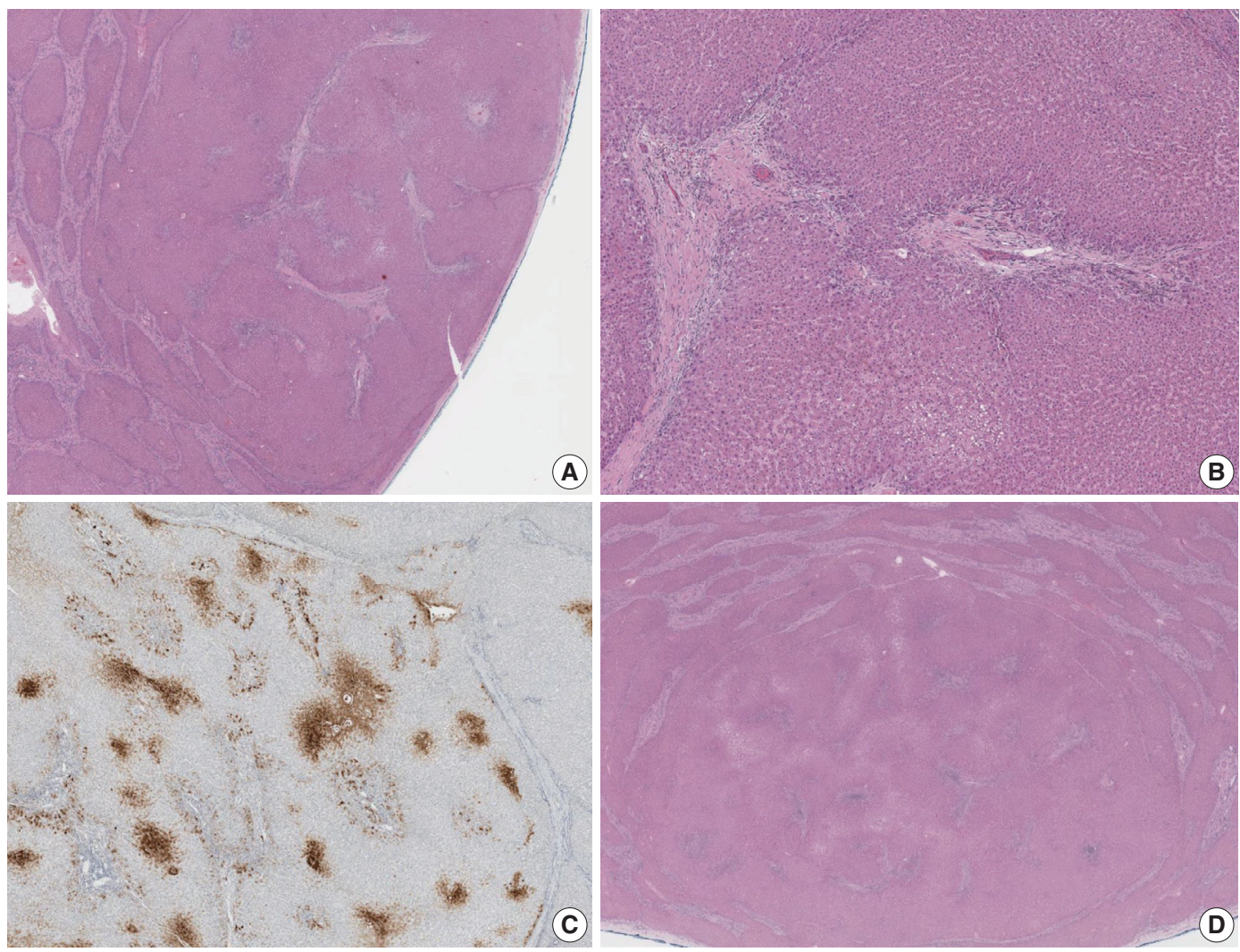

Fig. 3. Smaller focal nodular hyperplasia-like nodules were seen $(A-C)$ with small fibrous scars containing thick-walled blood vessels and patchy non-map-like glutamine synthetase expression. Fibrous septa were also seen in a large regenerative nodule but the central scar-like fibrous tissue was less prominent (D).

tures, such as steatosis and low risk of malignant transformation.

\section{Ethics Statement}

This case study was approved by the Institutional Review Board of Seoul National University Hospital (\#H-1809-141-975) and informed consent was waived.

\section{ORCID}

Yangkyu Lee https://orcid.org/0000-0002-6008-7713

Hyunjin Park https://orcid.org/0000-0001-7193-9849

Kyoungbun Lee https://orcid.org/0000-0001-8427-3003

Youngeun Lee https://orcid.org/0000-0002-0000-1615

Kiryang Lee https://orcid.org/0000-0003-3769-001X

Haeryoung Kim https://orcid.org/0000-0002-4205-9081

\section{Author Contributions}

Conceptualization: YL (Yangkyu Lee), HK. Funding acquisition: HK. Investigation: YL (Yangkyu Lee), HK. Supervision: HK. Writing-original draft: YL (Yangkyu Lee). Writing_review \& editing: YL (Yangkyu Lee), HP, KL, YL (Youngeun Lee), KL, HK. Approval of final manuscript: all authors.

\section{Conflicts of Interest}

H.K., a contributing editor of the Journal of Pathology and Translational Medicine, was not involved in the editorial evaluation or decision to publish this article. All remaining authors have declared no conflicts of interest.

\section{Funding Statement}

No funding to declare.

\section{References}

1. Bioulac-Sage P, Sempoux C, Balabaud C. Hepatocellular adenoma: classification, variants and clinical relevance. Semin Diagn Pathol 2017; 34: 112-25.

2. Nault JC, Paradis V, Cherqui D, Vilgrain V, Zucman-Rossi J. Molecular classification of hepatocellular adenoma in clinical practice. J Hepatol 2017; 67: 1074-83.

3. Rebouissou S, Imbeaud S, Balabaud C, et al. HNF1alpha inactivation promotes lipogenesis in human hepatocellular adenoma independently of SREBP-1 and carbohydrate-response element-binding protein (ChREBP) activation. J Biol Chem 2007; 282: 14437-46.

4. Ibarrola C, Castellano VM, Colina F. Focal hyperplastic hepatocellular nodules in hepatic venous outflow obstruction: a clinicopathological study of four patients and 24 nodules. Histopathology 2004; 44: 172-9.

5. Kondo F. Benign nodular hepatocellular lesions caused by abnormal hepatic circulation: etiological analysis and introduction of a new concept. J Gastroenterol Hepatol 2001; 16: 1319-28.

6. Sempoux C, Balabaud C, Paradis V, Bioulac-Sage P. Hepatocellular nodules in vascular liver diseases. Virchows Arch 2018; 473: 33-44.

7. Sempoux C, Paradis V, Komuta M, et al. Hepatocellular nodules expressing markers of hepatocellular adenomas in Budd-Chiari syn- 
drome and other rare hepatic vascular disorders. J Hepatol 2015; 63: 1173-80.

8. Sanada Y, Mizuta K, Niki T, et al. Hepatocellular nodules resulting from congenital extrahepatic portosystemic shunts can differentiate into potentially malignant hepatocellular adenomas. J Hepatobiliary Pancreat Sci 2015; 22: 746-56.
9. Bertheau P, Degott C, Belghiti J, et al. Adenomatous hyperplasia of the liver in a patient with congenital hepatic fibrosis. J Hepatol 1994; 20:213-7.

10. Fukushima N, Kuromatsu R, Uchiyama D, et al. Hyperplastic nodular hepatic lesions following end-to-side portacaval shunting in childhood. Intern Med 2007; 46: 1203-8. 